\title{
Chaos Suppression of an Electrically Actuated Microresonator Based on Fractional-Order Nonsingular Fast Terminal Sliding Mode Control
}

\author{
Jianxin Han, ${ }^{1}$ Qichang Zhang, ${ }^{2,3}$ Wei Wang, ${ }^{2,3}$ Gang Jin, ${ }^{1}$ Houjun Qi, ${ }^{1}$ and Qiu Li ${ }^{1}$ \\ ${ }^{1}$ Tianjin Key Laboratory of High Speed Cutting and Precision Machining, Tianjin University of Technology and Education, \\ Tianjin 300222, China \\ ${ }^{2}$ Department of Mechanics, School of Mechanical Engineering, Tianjin University, Tianjin 300072, China \\ ${ }^{3}$ Tianjin Key Laboratory of Nonlinear Dynamics and Control, Tianjin University, Tianjin 300072, China
}

Correspondence should be addressed to Qichang Zhang; qzhang@tju.edu.cn

Received 10 November 2016; Accepted 26 January 2017; Published 20 February 2017

Academic Editor: Oded Gottlieb

Copyright ( 2017 Jianxin Han et al. This is an open access article distributed under the Creative Commons Attribution License, which permits unrestricted use, distribution, and reproduction in any medium, provided the original work is properly cited.

\begin{abstract}
This paper focuses on chaos suppression strategy of a microresonator actuated by two symmetrical electrodes. Dynamic behavior of this system under the case where the origin is the only stable equilibrium is investigated first. Numerical simulations reveal that system may exhibit chaotic motion under certain excitation conditions. Then, bifurcation diagrams versus amplitude or frequency of AC excitation are drawn to grasp system dynamics nearby its natural frequency. Results show that the vibration is complex and may exhibit period-doubling bifurcation, chaotic motion, or dynamic pull-in instability. For the suppression of chaos, a novel control algorithm, based on an integer-order nonsingular fast terminal sliding mode and a fractional-order switching law, is proposed. Fractional Lyapunov Stability Theorem is used to guarantee the asymptotic stability of the system. Finally, numerical results with both fractional-order and integer-order control laws show that our proposed control law is effective in controlling chaos with system uncertainties and external disturbances.
\end{abstract}

\section{Introduction}

Microelectromechanical systems (MEMS) have been developed rapidly in the field of sensing and actuating [1-4]. However, as one of the most undesirable dynamic behaviors, chaotic motion exists extensively in dynamic MEMS, such as microresonators [5], comb-drivers [6], or atomic force microscopes (AFM) [7]. In many cases, the existence of chaos is highly undesirable as it can lead to poor performance $[8,9]$. Therefore, suppression of chaos of microcomponents has attracted much attention and is studied by many groups. For instance, applying Melnikov method, Ashhab et al. [10] derived the chaotic region of a microcantilever sample system with a proportional and derivative controller and finally eliminated the possibility of chaos. Yamasue and Hikihara [11] introduced a time delayed feedback control method to suppress the chaotic motion of a microcantilever sensor. An optimal linear control method was used to destroy the chaotic motion of a time-varying capacitor [12]. In the work by Wang et al. [9], a robust algorithm composed of sliding model control and a backstepping feedback was proposed and used to realize the suppression of chaos of an AFM system. Moreover, a nonlinear mechanical model for MEMS-based storage devices was developed and two control strategies: static output feedback control and geometric nonlinear control, were applied to eliminate the chaotic motion [13]. Tusset et al. [14] used three control strategies: state dependent Riccati equation control, optimal linear feedback control, and fuzzy sliding mode control, to sufficiently suppress the chaotic behavior of a comb-driver. Seleim et al. [15] investigated the dynamics of a close-loop controlled electrostatic MEMS resonator and finally determined the optimal operating regions beyond chaos.

Recently, chaotic motion of a doubly clamped microbeam actuated by two symmetrical electrodes was investigated by Haghighi and Markazi [16]. As the existences of cubic stiffness 
and electrostatic force, system may have different number of equilibrium points. They paid their attention to the vibration case where the origin is an unstable saddle point. Results showed that homoclinic bifurcation could lead to system chaos. Then, a robust adaptive fuzzy control algorithm was applied to control the chaotic motion and hence stabilize it into a high-amplitude oscillation state. What follows are some other control strategies, such as fuzzy sliding mode control [17] and second-order fast terminal sliding mode control [8], that were also used for the suppression of chaos. What is more, Aghababa [18] investigated the chaotic motion of a fractional-order model of this MEMS resonator and introduced a novel fractional finite-time controller to suppress the chaotic behavior with system uncertainty and external disturbance. Although a fractional-order MEMS system is chosen as a research model, this work indicates the possibility of applying fractional-order controllers to suppress chaotic motion in MEMS resonator.

Fractional calculus has a long history [19]. However, for many years, this branch of science had not been applied in physics and engineering. Until recent decades, the investigation of fractional calculus has been growing very rapidly, among which design of fractional-order controller has become an attractive research topic [20, 21]. Meanwhile, sliding mode control is an efficient approach in the application of linear or nonlinear control. For the foregoing reasons, there are more and more new control methods composed of fractional calculus and sliding mode control [22, 23], and their application fields have been gradually expanded from integerorder dynamic systems to fractional-order ones [24-26]. Besides, compared with traditional integer-order control, fractional-order control can offer more degrees of freedom to designers to meet a predefined set of performance criteria [27]. If the fractional-order selection is appropriate, it can exhibit faster convergence and stronger robustness than that of integer-order one [28].

So far, to the best of our knowledge, there is still no chaotic analysis of this doubly clamped microbeam-based resonator when system includes only one stable origin equilibrium. Although this vibration situation had been thought to have no chaotic motion under small disturbances [29], we found its chaos phenomenon as the increase of AC voltage during working pattern. Besides, there are fewer fractional-order sliding mode control results about chaos suppression of this MEMS resonator. In this paper, a simulation case where the origin is the only stable center is first carried out to investigate the existence of chaos. Then, bifurcation diagram is used to study the chaotic behavior nearby its primary resonance frequency. For the suppression of chaos, a fractional-order nonsingular fast terminal sliding mode control method (FNFTSMC), based on an integer-order nonsingular fast terminal sliding mode [30] and a fractional-order switching law [22], is proposed and, then, the effectiveness and robustness in controlling chaotic motion are investigated with system uncertainty and external and stochastic disturbances (usually existing in practice).

The structure of this paper is as follows. In Section 2, some basics of fractional calculus are briefly reviewed. In Section 3, dynamic model is introduced [16]. Chaotic analysis under the case where the origin is the only stable equilibrium is then carried out. In Section 4, a FNFTSNC method is proposed and its asymptotical stability is then proved via the Fractional Lyapunov Stability Theorem. In Section 5, simulation results are presented to verify the effectiveness of the proposed control scheme. Finally, Section 6 presents the conclusion.

\section{Some Basics of Fractional Calculus}

In this section, some basic definitions and preliminaries of fractional calculus are presented first. Then, a numerical approximation of fractional operators in the frequencydomain is given.

2.1. Definitions and Preliminaries. There is a uniform formula for the fractional integral of function $f(t)$ with $p$ th-order which is defined as [19]

$$
{ }_{t_{0}} I_{t}^{p} f(t)={ }_{t_{0}} D_{t}^{-p} f(t)=\frac{1}{\Gamma(p)} \int_{t_{0}}^{t} \frac{f(\xi)}{(t-\xi)^{1-p}} \mathrm{~d} \xi
$$

where $p>0, t_{0}$ and $t$ are the limits of the operation, and $\Gamma(\cdot)$ is the well-known Gamma function.

Two important and commonly used definitions of the fractional derivatives are the Riemann-Liouville and the Caputo fractional derivatives. In this paper, the RiemannLiouville definition is adopted and denoted as $D^{p}$, which is defined as [19]

$$
D^{p} f(t)=\frac{1}{\Gamma(m-p)} \frac{\mathrm{d}^{m}}{\mathrm{~d} t^{m}} \int_{t_{0}}^{t} \frac{f(\xi)}{(t-\xi)^{p-m+1}} \mathrm{~d} \xi
$$

where $m-1 \leq p<m, m \in \mathbb{N}$.

Property 1 (see [19]). For the Riemann-Liouville derivative, the following equality holds:

$$
\begin{aligned}
D^{p}\left[D^{q} f(t)\right]= & D^{q+p}[f(t)] \\
& -\sum_{j=1}^{k}\left[D^{q-j} f(t)\right]_{t=t_{0}} \frac{\left(t-t_{0}\right)^{-p-j}}{\Gamma(1-p-j)},
\end{aligned}
$$

where $p>0,0 \leq k-1 \leq q<k, k \in \mathbb{N}$.

Property 2 (see [31]). The following equality holds for the Riemann-Liouville derivative:

$$
D^{-p} f\left(t_{0}\right)=0
$$

where $p>0$.

Property 3. According to Properties 1 and 2, if $p>0$ and $0<$ $q<1$, one has

$$
D^{p}\left[D^{q} f(t)\right]=D^{q+p}[f(t)] .
$$

Property 4 (see [22]). If $0<p<1$, one has

$$
\operatorname{sgn}\left[D^{p} \operatorname{sgn}(f)\right]=\operatorname{sgn}(f),
$$

where $\operatorname{sgn}(\cdot)$ is the sign function. 
Theorem 1 (see $[26,32,33])$. Let $x=0$ be an equilibrium point for the nonautonomous fractional-order system

$$
D^{p} x(t)=f(x, t)
$$

where $f(x, t)$ satisfies the Lipschitz condition with Lipschitz constant $l>0$ and $p \in(0,1)$. Assume that there exists a Lyapunov function $V(t, x)$ satisfying

$$
\begin{aligned}
\alpha_{1}\|x\|^{a} & \leq V(t, x) \leq \alpha_{2}\|x\|, \\
\frac{\mathrm{d} V(t, x)}{\mathrm{d} t} & \leq-\alpha_{3}\|x\|,
\end{aligned}
$$

where $\alpha_{1}, \alpha_{2}, \alpha_{3}$, and a are positive constants and $\|\cdot\|$ denotes an arbitrary norm. Then the equilibrium point of system (7) is asymptotically stable.

2.2. Numerical Approximation. In practice, it is difficult to obtain the exact solution of fractional differential equations and, in most cases, $f(t)$ is unknown. These make numerical approximations be widely used. Among these numerical methods, Oustaloup approximation is one of the most commonly used ways to obtain fractional operators [34]. In 2006, Xue et al. [35] proposed a refined Oustaloup filter, which has a very good approximation of the fractional operators in a specified frequency range $\left(\omega_{b}, \omega_{h}\right)$ of order $N$. This filter is given by

$$
s^{v} \approx\left(\frac{d \omega_{h}}{b}\right)^{v}\left[\frac{d s^{2}+b \omega_{h} s}{d(1-v) s^{2}+b \omega_{h} s+d v}\right] \prod_{k=-N}^{N} \frac{s+\omega_{k}^{\prime}}{s+\omega_{k}}
$$

where $v$ is the order of fractional operation; $\omega_{k}$ and $\omega_{k}^{\prime}$ can be computed from

$$
\begin{aligned}
& \omega_{k}=\left(\frac{b \omega_{h}}{d}\right)^{(v+2 k) /(2 N+1)}, \\
& \omega_{k}^{\prime}=\left(\frac{d \omega_{b}}{b}\right)^{(v-2 k) /(2 N+1)},
\end{aligned}
$$

where $b$ and $d$ are weighted parameters. It is expected that a good approximation is obtained with $b=10$ and $d=9$.

\section{System Description}

As is shown in Figure 1, an electrostatically actuated microresonator is simplified as a one degree of freedom system [16]. $\widehat{x}$ is the vertical displacement of the microbeam; $d_{0}$ is the initial gap width. The resonator is deflected by DC voltage $V_{\mathrm{dc}}$ and actuated by AC voltage $V_{\mathrm{ac}} \sin (\Omega t)$, where $V_{\mathrm{dc}}$ and $V_{\mathrm{ac}}$ are the amplitudes of DC and AC voltages and $\Omega$ is the angular frequency of AC voltage. Displacement and velocity of this resonator can be identified through the measurement of output voltage $V_{\text {out }}$ in practice [36].

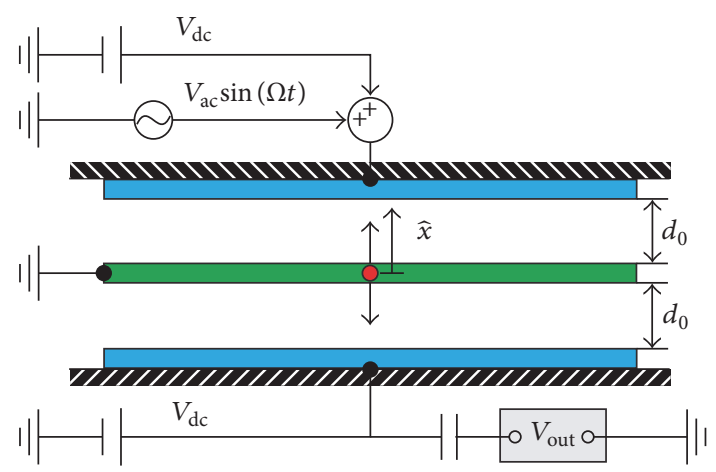

FIGURE 1: Schematic diagram of an electrostatically actuated microresonator.

The electrostatic force $F_{e}$ loaded on the microresonator can be expressed as [36]

$$
\begin{aligned}
F_{e}= & \frac{1}{2} \frac{C_{0} d_{0}}{\left(d_{0}-\hat{x}\right)^{2}}\left[V_{\mathrm{dc}}+V_{\mathrm{ac}} \sin (\Omega t)\right]^{2} \\
& -\frac{1}{2} \frac{C_{0} d_{0}}{\left(d_{0}+\widehat{x}\right)^{2}} V_{\mathrm{dc}}^{2}
\end{aligned}
$$

where $C_{0}=\varepsilon_{d} A_{s} / d_{0}$ is the capacitance over the gap when $\hat{x}=0, \varepsilon_{d}$ is the dielectric constant of the air, and $A_{s}$ is the overlapped area between the microbeam and the fixed electrode. The governing equation of motion of the resonator can be expressed as [16]

$$
m \widehat{x}^{\prime \prime}+c \widehat{x}^{\prime}+k_{1} \widehat{x}+k_{3} \widehat{x}^{3}=F_{e},
$$

where the prime represents the derivative with respect to time $t$ and $m, c, k_{1}$, and $k_{3}$ are the effective mass and damping, linear, and cubic stiffness, respectively.

For convenience, one can introduce the following nondimensional variables:

$$
\begin{aligned}
\tau & =\omega_{0} t, \\
x & =\frac{\widehat{x}}{d_{0}}, \\
\mu & =\frac{c}{\sqrt{k_{1} m}}, \\
\alpha & =\frac{k_{3} d_{0}^{2}}{k_{1}}, \\
\gamma & =\frac{C_{0} V_{\mathrm{dc}}^{2}}{2 k_{1} d_{0}^{2}}, \\
\rho & =\frac{V_{\mathrm{ac}}}{V_{\mathrm{dc}}}, \\
\omega & =\frac{\Omega}{\omega_{0}},
\end{aligned}
$$




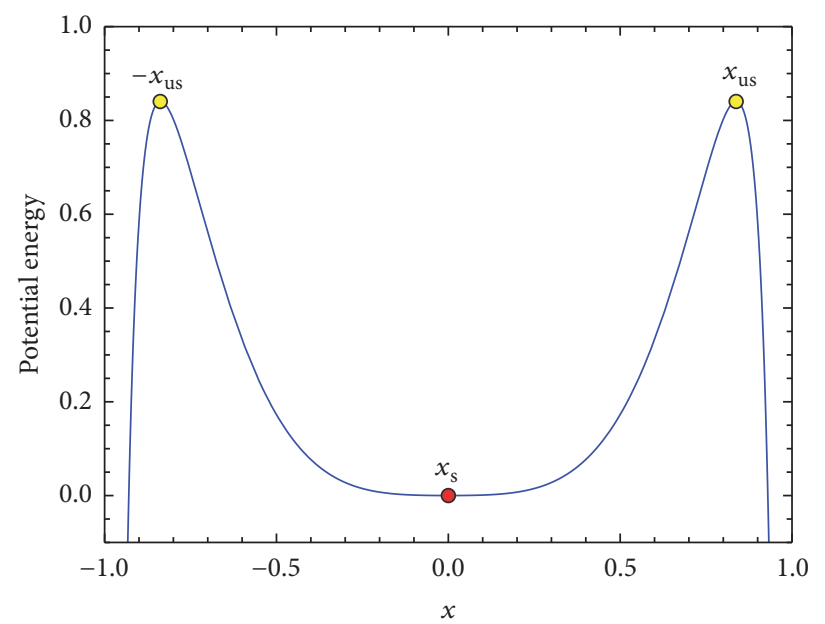

(a)

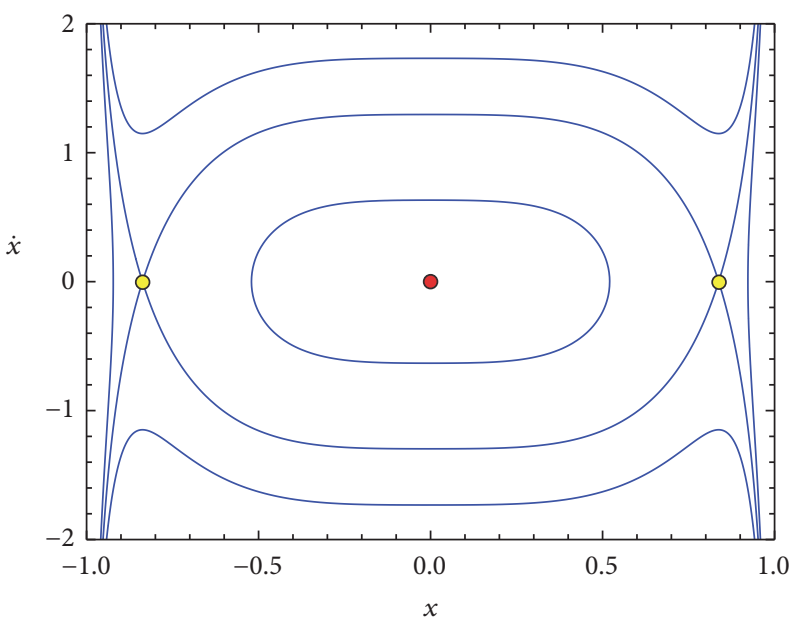

(b)

Figure 2: Potential energy (a) and phase portrait (b) of Hamiltonian system of (14).

where $\omega_{0}=\sqrt{k_{1} / m}$ is the natural angular frequency of the microbeam. Then, (12) can be rewritten in the following nondimensional form (without any simplification):

$$
\begin{aligned}
\ddot{x}+\mu \dot{x}+x+\alpha x^{3}= & \gamma\left[\frac{1}{(1-x)^{2}}-\frac{1}{(1-x)^{2}}\right] \\
& +\frac{2 \gamma \rho}{(1-x)^{2}} \sin (\omega \tau) \\
& +\frac{\gamma \rho^{2}}{2(1-x)^{2}}[1-\cos (2 \omega \tau)],
\end{aligned}
$$

where the overdot represents the derivative with respect to nondimensional time $\tau$.

In our previous study $[37,38]$, we find that the number of equilibrium points of this system is determined by both $\alpha$ and $\gamma$. Under the situation with $\alpha>2$, this system has only one stable center at the origin when $0<\gamma<0.25$ and two stable centers at either side of the origin when $0.25<\gamma<(1+$ $\alpha)^{3} /\left(27 \alpha^{2}\right)$. Taking $\alpha=12$ as an example, the system has two stable centers when $\gamma=0.338$. Under some certain AC excitations, system may exhibit chaotic motion [16]. In this paper, $0<\gamma<0.25$ is considered here to investigate the chaotic behavior of this microresonator when system only has one stable origin center.

Numerical simulations are carried out as follows. Nondimensional parameters are assumed to be $\alpha=12, \gamma=0.21$, and $\mu=0.005$. Potential energy and phase portrait of Hamiltonian system of (14) are shown in Figure 2. In this case, system contains only one stable origin equilibrium point $x_{s}=0$. Besides, two symmetrical unstable equilibrium points $\pm x_{\mathrm{us}}=$ \pm 0.837446 are located at either side of it. Theoretical maximum vibration amplitude is approximate to $x_{\mathrm{us}}$. Generally, microresonator is driven to vibrate around its natural frequency $[39,40]$. Through some calculation, one can derive that the linearized equivalent natural angular frequency of this microresonator is $\omega_{n}=\sqrt{1-4 \gamma}$ in nondimensional form. Therefore, AC excitation angular frequency $\omega$ should be selected in the neighborhood of $\omega_{n}$. For case study, excitation parameters are selected as $\rho=0.33$ and $\omega=0.4$. Numerical solutions of system (14) are calculated by removing 5000 periods $(T=2 \pi / \omega)$ of AC excitation and retaining the last 128 periods. Initial conditions are assumed to be $(0,0)$. Finally, phase portrait and time history diagram of (14) are shown in Figure 3. The red lines in Figure 3(a) are two heteroclinic orbits, representing stable and unstable boundary. Inner region is stable beyond pull-in instability. In Figure 3(b), two red lines indicate the available motion region of the microresonator. From Figure 3, one can notice that system vibration is quite complex. Detailed numerical analyses (Figure 4) via Poincare maps, power spectral density (PSD), and maximum Lyapunov exponents (MLE) demonstrate that the microresonator indeed undergoes chaotic motion. Thus, system (14) may exhibit complex dynamics such as chaos during working pattern (namely, the resonator is excited near its natural frequency).

In what follows, bifurcation diagram is drawn to analyze the chaotic behavior of this resonator, which is obtained based on Poincare section taken by sampling the data at each period of AC excitation. We consider the following system parameters [41]: $m=5 \times 10^{-12} \mathrm{~kg}, k_{1}=5 \mu \mathrm{N} / \mu \mathrm{m}, k_{3}=$ $15 \mu \mathrm{N} / \mu \mathrm{m}^{3}, d_{0}=2 \mu \mathrm{m}$, and $C_{0}=1.5 \times 10^{-14} \mathrm{~F}$. A quality factor is assumed to be $Q=200$, which is related to the damping coefficient by $c=\sqrt{k_{1} m} / Q$ [40]. Taking $V_{\mathrm{dc}}=23.66 \mathrm{~V}$ yields $\gamma \approx 0.21$. Then, the dimensional equivalent natural angular frequency is approximate to $4 \times 10^{5} \mathrm{rad} / \mathrm{s}(63.66 \mathrm{kHz})$. Therefore, the following analysis mainly focuses on the vibration nearby its natural frequency.

In Figure 5, dynamic behavior of the system is depicted as the variation of $\mathrm{AC}$ voltage amplitude. As the increase of $V_{\mathrm{ac}}$, the resonator exhibits complex dynamics like Period 2 motion, Period 3 motion, period-doubling bifurcation, chaotic motion and so on. When $7.59 \mathrm{~V} \leq V_{\mathrm{ac}} \leq 8.42 \mathrm{~V}$, the system is almost chaotic. When $V_{\mathrm{ac}}>8.92 \mathrm{~V}$, dynamic pullin occurs. Next, the effect of frequency of AC excitation on chaotic motion is studied. Taking $V_{\mathrm{ac}}=8.5 \mathrm{~V}$ yields a Period 


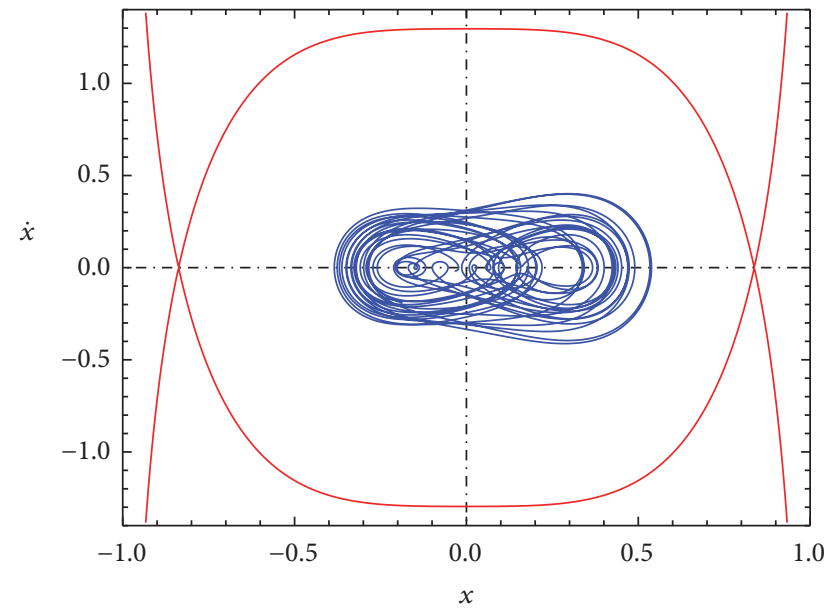

(a)

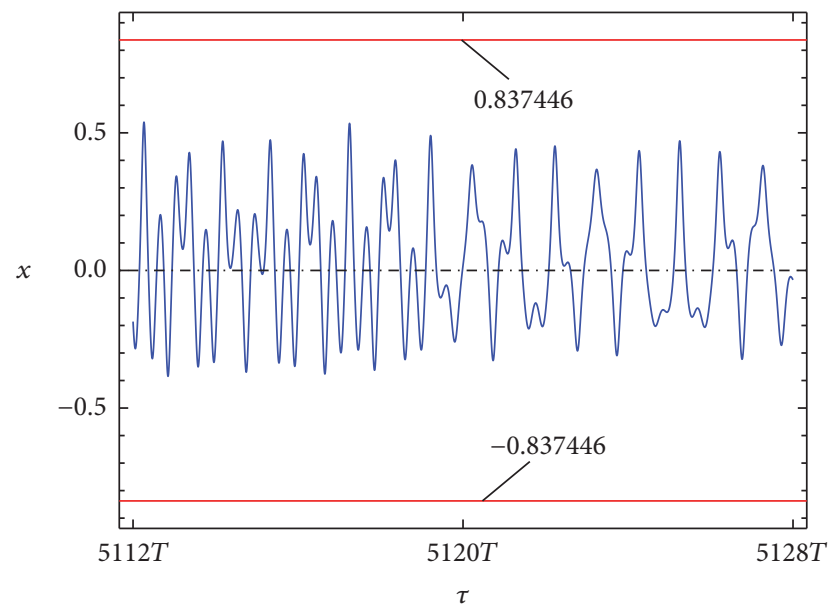

(b)

FIgURe 3: Phase portrait (a) and time history diagram (b) of dynamic system (14).

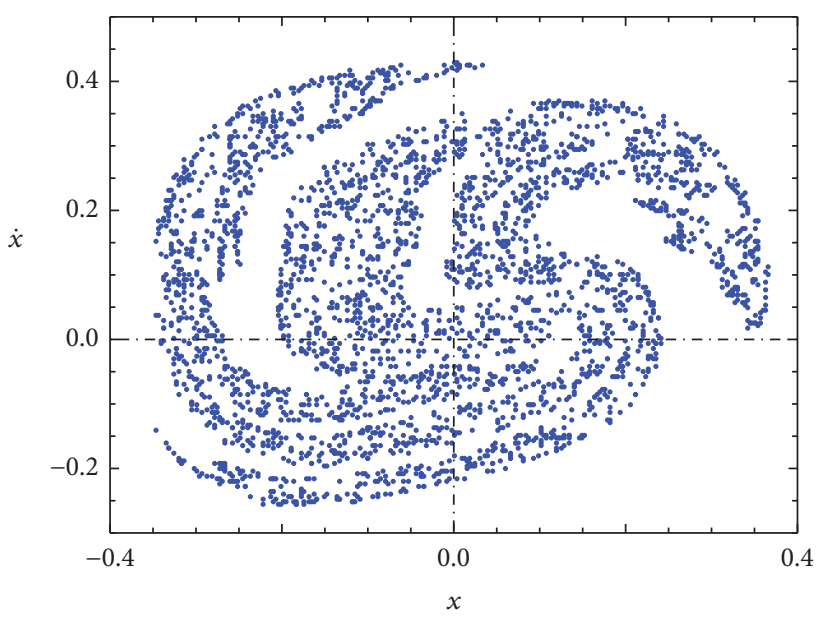

(a)

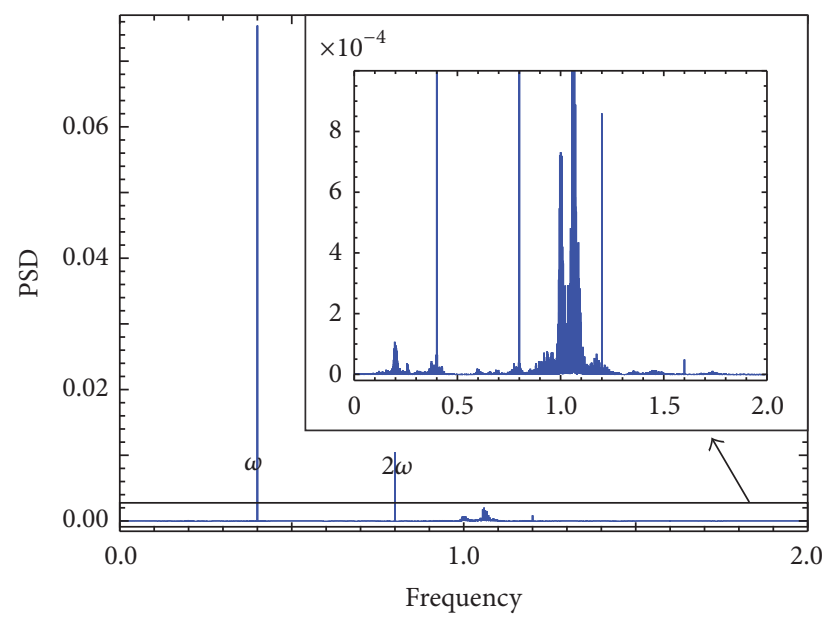

(b)

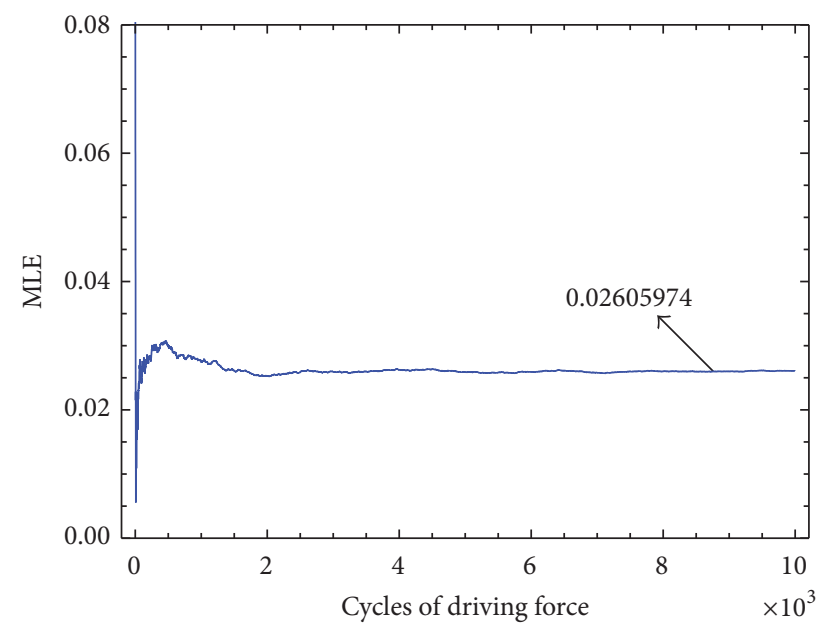

(c)

Figure 4: Poincare maps (a), power spectral density (PSD) (b), and maximum Lyapunov exponent (MLE) (c) of dynamic system (14). 


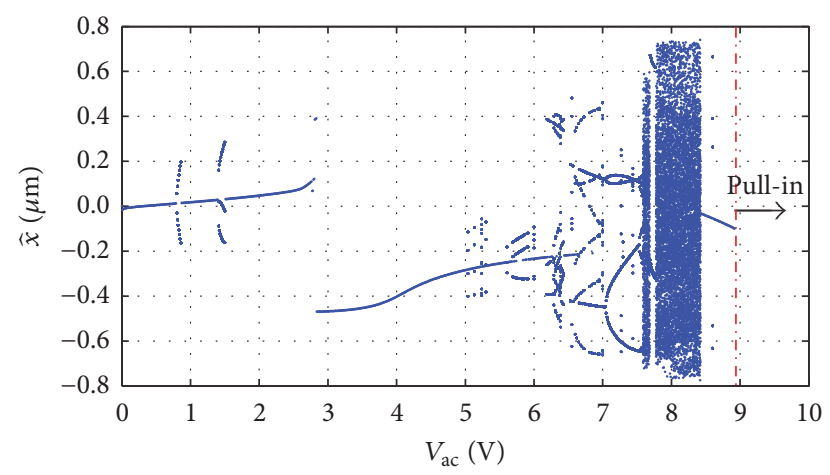

FIGURE 5: Bifurcation diagram versus the amplitude of AC voltage with $\Omega=4 \times 10^{5} \mathrm{rad} / \mathrm{s}$.

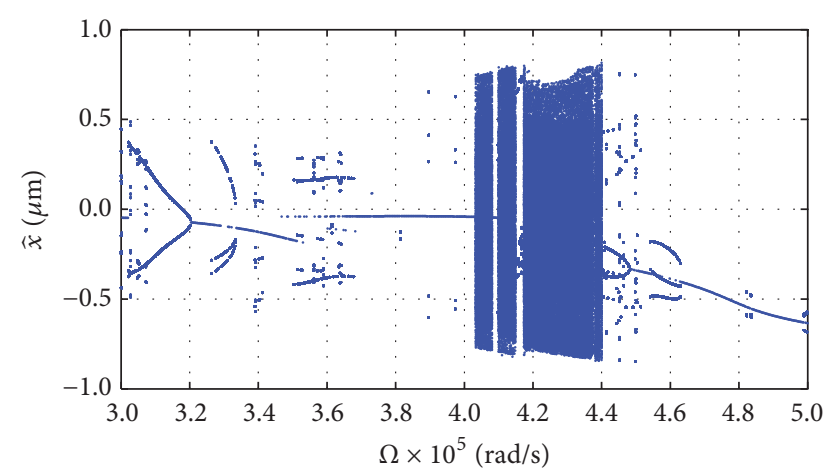

FIGURE 6: Bifurcation diagram versus the angular frequency of AC voltage with $V_{\mathrm{ac}}=8.5 \mathrm{~V}$.

1 motion (Figure 5). In Figure 6, the vibration characteristic is complex as well as when the angular frequency of $\mathrm{AC}$ voltage varies from $3 \times 10^{5} \mathrm{rad} / \mathrm{s}(47.75 \mathrm{kHz})$ to $5 \times 10^{5} \mathrm{rad} / \mathrm{s}$ $(79.58 \mathrm{kHz})$, especially between $4 \times 10^{5} \mathrm{rad} / \mathrm{s}(63.66 \mathrm{kHz})$ and $4.4 \times 10^{5} \mathrm{rad} / \mathrm{s}(70.03 \mathrm{kHz})$. When $\Omega$ varies from $4.03 \times$ $10^{5} \mathrm{rad} / \mathrm{s}$ to $4.12 \times 10^{5} \mathrm{rad} / \mathrm{s}$, system dynamic changes between Period 1 and chaotic motion. In domain $\left[4.2 \times 10^{5} \mathrm{rad} / \mathrm{s}, 4.4\right.$ $\times 10^{5} \mathrm{rad} / \mathrm{s}$, system is always chaotic.

The above analysis indicates that nearby the natural frequency, the vibration of the system is complex and may exhibit chaotic motion. In the following section, a FNFTSMC method is proposed to cope with this chaotic behavior.

\section{Control Method}

Consider the following integer-order system:

$$
\begin{aligned}
& \dot{x}=y, \\
& \dot{y}=f(x, y, \tau)+\Delta f(x, y, \tau)+d_{s}(\tau)+u(\tau),
\end{aligned}
$$

where $f$ is a known function, $\Delta f$ and $d_{s}$ are uncertainty term and external disturbance, and $u$ is the presented control law.
If the tracking errors of the controlled system are defined as $e_{1}=x-x_{d}$ and $e_{2}=y-\dot{x}_{d}$, where $x_{d}$ is the desired trajectory, then the error-dynamic system can be given as

$$
\begin{aligned}
\dot{e}_{1}= & e_{2}, \\
\dot{e}_{2}= & f\left(e_{1}+x_{d}, e_{2}+\dot{x}_{d}, \tau\right)+\Delta f\left(e_{1}+x_{d}, e_{2}+\dot{x}_{d}, \tau\right) \\
& +d_{s}(\tau)+u(\tau)-\ddot{x}_{d} .
\end{aligned}
$$

Assumption 2. Uncertainty $\Delta f$ and disturbance $d_{s}$ are usually unknown and assumed to be bounded as follows:

$$
\left\|\Delta f+d_{s}\right\| \leq M
$$

where $M$ is a positive constant.

Sliding mode control design usually consists of two steps: (a) sliding surface design; (b) effective control law design. In the first step, a uniform integer-order sliding surface $\sigma$ is introduced as follows [30]:

$$
\sigma=e_{1}+\kappa_{1}\left|e_{1}\right|^{\delta_{1}} \operatorname{sgn}\left(e_{1}\right)+\kappa_{2}\left|e_{2}\right|^{\delta_{2}} \operatorname{sgn}\left(e_{2}\right),
$$

where $\kappa_{1}$ and $\kappa_{2}$ are positive constants, $1<\delta_{2}<2, \delta_{1}>\delta_{2}$.

Remark 3. Expression (18) does not have singularity problem and, for any given initial condition $e_{1}(0)=e_{0}$, the system state converges to $e_{1}=0$ in finite time [30].

Sliding mode control law can be decomposed into two parts, equivalent control law and switching law. The former one, deduced according to predesigned sliding surface by the application of sliding mode control theory, guarantees system state on sliding surface, while the latter one constrains the state to sliding surface subsequently. Firstly, without any consideration of uncertainty and disturbance, the equivalent control law can be given as

$$
\begin{aligned}
u_{\mathrm{eq}}(\tau)= & \ddot{x}_{d}-f \\
& -\frac{1}{\kappa_{2} \delta_{2}}\left|e_{2}\right|^{2-\delta_{2}} \operatorname{sgn}\left(e_{2}\right)\left(1+\kappa_{1} \delta_{1}\left|e_{1}\right|^{\delta_{1}-1}\right) .
\end{aligned}
$$

Next, two different forms of switching law will be discussed as follows.

4.1. Integer-Order Nonsingular Fast Terminal Sliding Mode Control (INFTSMC). A traditional integer-order switching law can be described as [30]

$$
u_{r, 1}=-K_{1} \sigma-\left(K_{2}+\delta\right) \operatorname{sgn}(\sigma),
$$

where $K_{1}>0, K_{2}>0, \delta>0$.

Theorem 4 (see [30]). If control law $u_{1}=u_{e q}+u_{r, 1}$ is applied to the error-dynamic system (16), then the tracking error will converge to zero in finite time if $\delta>M$.

Proof. Consider the smooth Lyapunov function as

$$
V_{1}=\frac{1}{2} \sigma^{2}
$$


Taking the derivative of (21) with respect to time $\tau$ and using (16) and (18), one has

$$
\begin{aligned}
& \dot{V}_{1}=\sigma \dot{\sigma} \\
& =\kappa_{2} \delta_{2}\left|e_{2}\right|^{\delta_{2}-1}\left\{(\Delta f+d) \sigma-\left[K_{1} \sigma^{2}+\left(K_{2}+\delta\right)|\sigma|\right]\right\} \\
& \leq \kappa_{2} \delta_{2}\left|e_{2}\right|^{\delta_{2}-1}\left[(\|\Delta f+d\|-\delta)|\sigma|-K_{2}|\sigma|-K_{1} \sigma^{2}\right] \\
& <-\kappa_{2} \delta_{2}\left|e_{2}\right|^{\delta_{2}-1}\left(K_{1} \sigma^{2}+K_{2}|\sigma|\right) \leq 0 .
\end{aligned}
$$

Consequently, the tracking error will converge to zero.

\subsection{Fractional-Order Nonsingular Fast Terminal Sliding Mode} Control (FNFTSMC). Before introducing the fractionalorder switching law, a fractional-order reaching law must be described in advance. The expression of reaching law can be given by the following [22]:

$$
D^{1-\lambda} \sigma=-K_{1} \sigma-K_{2} \operatorname{sgn}(\sigma)
$$

where $0<\lambda<1$.

According to Property 3, the following operation holds:

$$
D^{\lambda}\left(D^{1-\lambda} \sigma\right)=D^{\lambda}\left[-K_{1} \sigma-K_{2} \operatorname{sgn}(\sigma)\right] .
$$

The above equation is equivalent to

$$
\dot{\sigma}=-D^{\lambda}\left[\left(K_{1}|\sigma|+K_{2}\right) \operatorname{sgn}(\sigma)\right] .
$$

Due to $0<\lambda<1, \operatorname{sgn}\left[D^{\lambda} \operatorname{sgn}(\sigma)\right]=\operatorname{sgn}(\sigma)$. The reaching dynamics governed by (25) will create a stronger push from both sides of the switching manifold [22].

According to the above analysis, a fractional-order switching law can be proposed and defined as

$$
u_{r, 2}=-D^{\lambda}\left[\left(K_{1}|\sigma|+K_{2}\right) \operatorname{sgn}(\sigma)\right]-\delta \operatorname{sgn}(\sigma),
$$

where $0<\lambda<1$.

In our study, a FNFTSMC method based on an integerorder sliding mode and a fractional-order switching law is proposed. The feasibility will be discussed as follows.

Theorem 5. Fractional-order control law $u_{2}=u_{e q}+u_{r, 2}$ is effective in controlling system (16), if $\delta>M$.

Although the error-dynamic system (16) is integer-order, the proposed sliding mode control is fractional-order. Therefore, it is more suitable to prove the occurrence of the sliding motion via the Fractional Lyapunov Stability Theorem (Theorem 1) [41].

Proof. Select a nonsmooth Lyapunov function as

$$
V_{2}=|\sigma| \text {. }
$$

Then, one has

$$
\begin{aligned}
\dot{V}_{2} & =\dot{\sigma} \operatorname{sgn}(\sigma)=\kappa_{2} \delta_{2}\left|e_{2}\right|^{\delta_{2}-1}\{(\Delta f+d) \\
& \left.-D^{\lambda}\left[\left(K_{1}|\sigma|+K_{2}\right) \operatorname{sgn}(\sigma)\right]-\delta \operatorname{sgn}(\sigma)\right\} \operatorname{sgn}(\sigma) \\
& \leq \kappa_{2} \delta_{2}\left|e_{2}\right|^{\delta_{2}-1}[\|\Delta f+d\|-\delta]-\kappa_{2} \delta_{2}\left|e_{2}\right|^{\delta_{2}-1} \\
& \cdot D^{\lambda}\left[\left(K_{1}|\sigma|+K_{2}\right) \operatorname{sgn}(\sigma)\right] \operatorname{sgn}(\sigma) .
\end{aligned}
$$

According to Property 4 and (25), one has the following [22]:

$$
D^{\lambda}\left[\left(K_{1}|\sigma|+K_{2}\right) \operatorname{sgn}(\sigma)\right] \operatorname{sgn}(\sigma) \geq 0 .
$$

Then (28) can be simplified as

$$
\dot{V}_{2} \leq-\kappa_{2} \delta_{2}\left|e_{2}\right|^{\delta_{2}-1}[\delta-\|\Delta f+d\|] .
$$

As the sliding surface is convergent [30], it is reasonable to assume that $\sigma$ is bounded in $\|\sigma\| \leq \Lambda$, where $\Lambda$ is a positive constant. Then, (30) can be written as

$$
\begin{aligned}
\dot{V}_{2} & \leq-\kappa_{2} \delta_{2}\left|e_{2}\right|^{\delta_{2}-1}[\delta-\|\Delta f+d\|] \\
& \leq-\kappa_{2} \delta_{2}\left|e_{2}\right|^{\delta_{2}-1}[\delta-\|\Delta f+d\|] \frac{\|\sigma\|}{\Lambda} .
\end{aligned}
$$

Therefore, $V_{2}$ satisfies fractional-order Lyapunov stability theorem (Theorem 1). The proof is completed.

Remark 6 (see [42]). Nonsmooth Lyapunov functions can be applied to show the finite-time convergence of a system.

The signum function of the switching law is one main inducement of chattering. When the system state hits the sliding surface, chattering will occur because of the discontinuity of signum function. To reduce chattering, a saturation function is often used instead of signum function. Here, a new form of saturation function is introduced as follows [43]:

$$
\operatorname{sat}\left[\frac{\operatorname{sig}(\sigma)^{\varphi}}{\varepsilon_{1}^{\varphi}}\right]= \begin{cases}\frac{\operatorname{sig}(\sigma)^{\varphi}}{\left\|\operatorname{sig}(\sigma)^{\varphi}\right\|}, & \|\sigma\|>\varepsilon_{1} \\ \frac{\operatorname{sig}(\sigma)^{\varphi}}{\varepsilon_{1}^{\varphi}}, & \|\sigma\| \leq \varepsilon_{1},\end{cases}
$$

where $\operatorname{sig}(\sigma)^{\varphi}=|\sigma|^{\varphi} \operatorname{sgn}(\sigma), \varepsilon_{1}$ is the boundary layer, and $0<\varphi<1$.

\section{Simulation Results}

In order to demonstrate the effectiveness of the proposed control method, simulation results are provided in this section. Numerical simulations based on (14) are carried out using the fourth-order Runge-Kutta method with 0.001 time-step size.

Control flow chart is shown in Figure 7. Error-dynamic signals $\left(e_{1}\right.$ and $\left.e_{2}\right)$ can be calculated through the comparison of system signals $(x$ and $\dot{x})$ and desired trajectories $\left(x_{d}\right.$ and $\left.\dot{x}_{d}\right)$. Then, fractional-order controller $u_{\mathrm{eq}}+u_{r, 2}$ can be obtained through two steps. Integer-order operator can be used to calculate equivalent control law (19) and fractional-order switching law (26) can be calculated through a fractionalorder filter (9). Meanwhile, traditional integer-order controller $u_{\mathrm{eq}}+u_{r, 1}$ can be calculated just through an integerorder operator. In practice, displacement and velocity signals of the microresonator can be obtained by $V_{\text {out }}$ transformation. Through calculation, control $u$ can now be obtained and loaded on this microresonator in the form of external force, like electrostatic force (electrode is acted by feedback voltage), piezoresponse force (microbeam attached by piezoelectric layers), and so forth. As our main focus is on the 


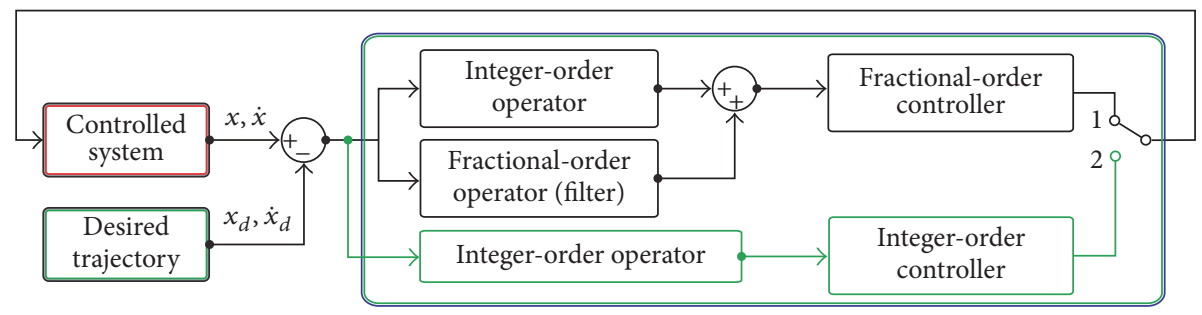

FIGURE 7: Control flow chart.

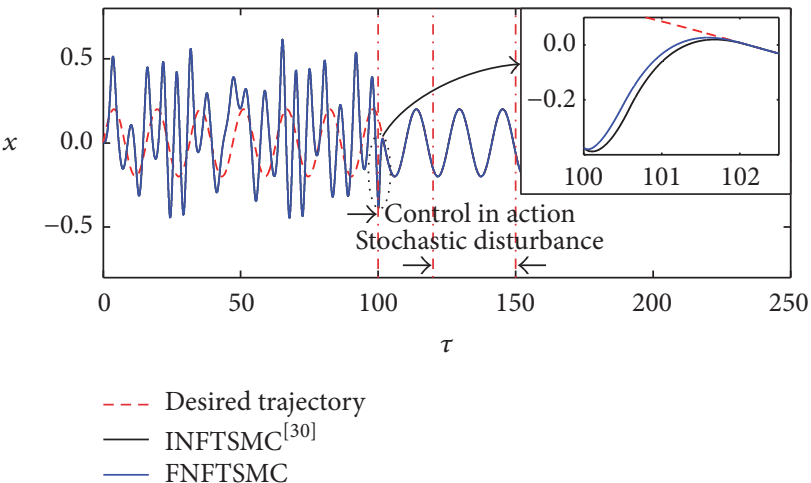

(a)

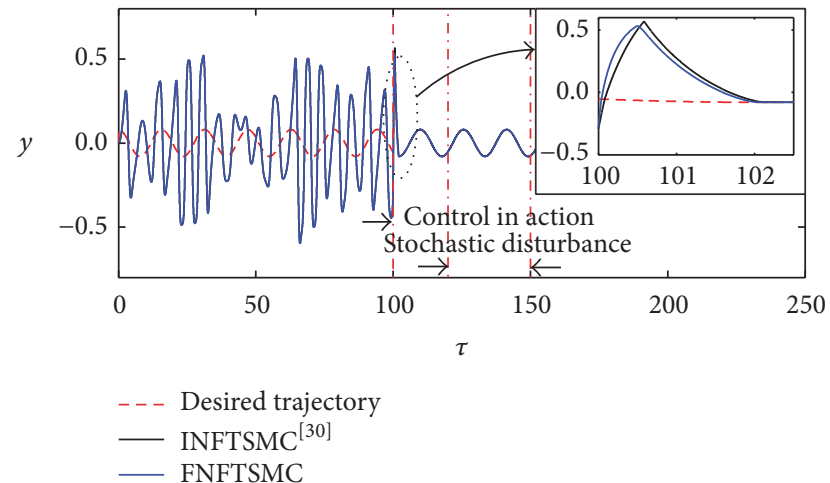

(b)

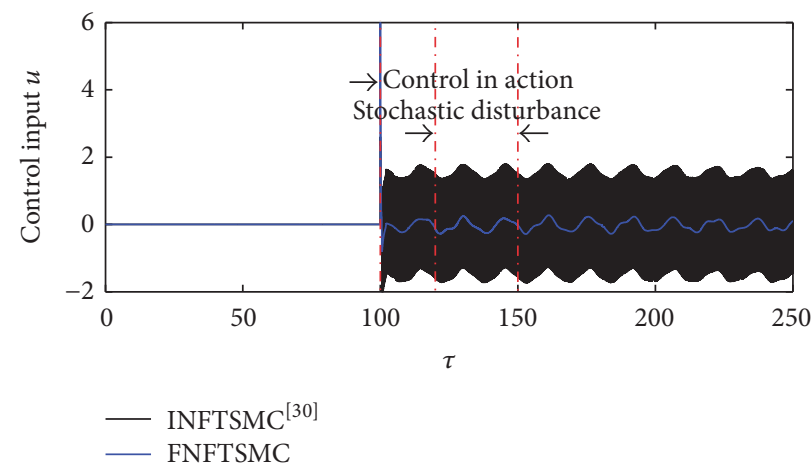

(c)

FIGURE 8: Simulation results in Case 1 under different control strategies: (a) displacement response; (b) velocity response; (c) control input.

control strategy design and its application in the field of chaos suppression, detailed implementation is not discussed here anymore.

Assume $V_{\mathrm{ac}}=7.7 \mathrm{~V}$ and $\Omega=4.2 \times 10^{5} \mathrm{rad} / \mathrm{s}$; other parameters are the same as Section 3. Under these parameters, the resonator evolves into chaotic state. The control target is to let the motion of the resonator track the desired trajectory $x_{d}=$ $0.2 \sin (0.4 \tau)$. In practice, system uncertainties and external disturbances are usually the most common disturbances in MEMS. Thus, to verify the effectiveness and robustness of the proposed method, the uncertainty and disturbance are assumed to be as follows: (a) Case 1: $\Delta f=0.2 \sin \left(\sqrt{x^{2}+y^{2}}\right)$ and $d_{s}=0.05 \sin \tau$; (b) Case $2: \Delta f=0.2 \Re$ and $d_{s}=0.05 \Re$, where stochastic parameter $\mathfrak{R}$ follows the standard uniform distribution on the open interval $(0,1)$. The controller is activated at $\tau=100$. Besides, a small stochastic disturbance $d_{r}$ is added onto the system when $\tau \in[120,150] . d_{r}$ follows the standard uniform distribution on the open interval $(0,0.05)$. Control parameters in these two cases are given as $b=10, d=$ $9, \omega_{b}=10^{-3}, \omega_{h}=10^{3}, N=9, \delta=0.5, \kappa_{1}=1, \kappa_{2}=1, \delta_{1}=2$, $\delta_{2}=5 / 3, K_{1}=1, K_{2}=1, \varphi=1 / 3, \varepsilon_{1}=0.05$, and $\lambda=0.3$.

Simulation results are shown in Figures 8 and 9 . The black line represents the trajectory of the traditional INFTSMC with signum function switching law [30], and the blue line represents the trajectory of our proposed FNFTSMC with novel saturation function switching law. From these two figures, one can notice that

(1) compared with the control method by L. Yang and J. Yang [30], our proposed control method is effective in eliminating the chaotic motion;

(2) under this set of control parameters, the trajectories of the proposed FNFTSMC converge slightly faster than that of INFTSMC; 


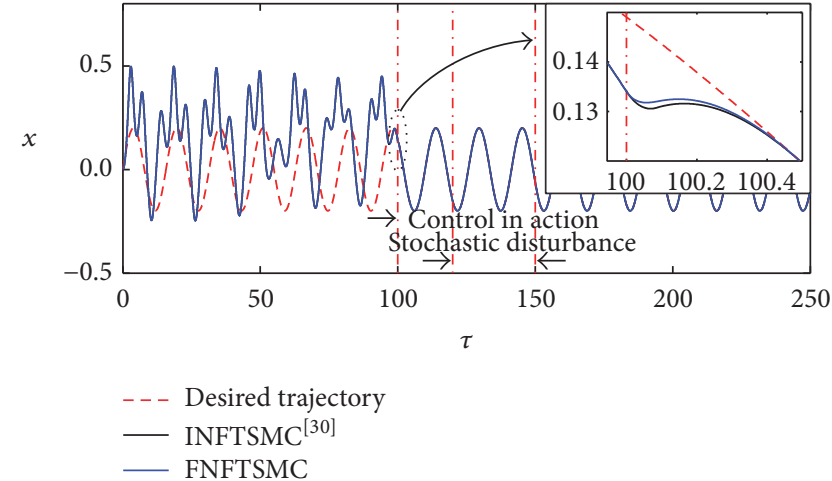

(a)

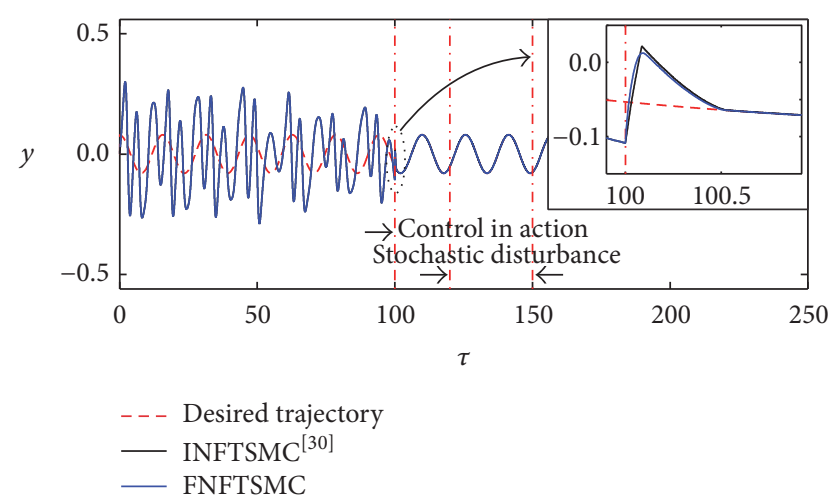

(b)

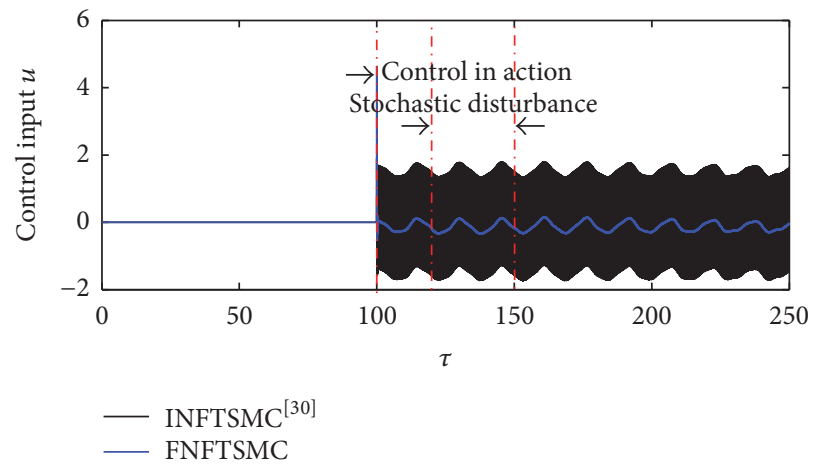

(c)

FIGURE 9: Simulation results in Case 2 under different control strategies: (a) displacement response; (b) velocity response; (c) control input.

(3) the novel saturation function is effective in reducing chattering;

(4) the proposed method has strong robustness to the system (random) uncertainty and disturbance.

Appropriate fractional-order selection can make FNFTSMC exhibit faster convergence and stronger robustness [22]. Therefore, our next focus is mainly on a qualitative discussion about the effect of fractional-order on control performance.

Standard deviation can reflect the degree of dispersion of a data set, so this statistic can be used to describe the degree of chattering of control signal $u$. The larger the standard deviation of input is, the more dramatic the chattering is. Meanwhile, a nondimensional statistical function can be defined to approximately estimate the control energy cost. It should be noticed that no matter what the sign of the work done by the controller is, the control system is always energy-intensive. Thus, a mean power to describe the energy cost can be defined as

$$
P=\frac{1}{T_{c}} \sum_{i=S t}^{E n-1}\left|u_{i}\left(x_{i+1}-x_{i}\right)\right|
$$

where $T_{c}$ represents the total calculated time from control in action to the end, $S t$ is the discrete point number when control is on, and $E n$ is equal to the total length of discrete points. When control is activated, control signal $u_{S t}$ can be calculated according to the difference between the actual trajectory $x_{S t}$ and the desired trajectory $x_{d, S t}$. Under the effect of excitation and control input $u_{S t}$, the trajectory can be driven from $x_{S t}$ to $x_{S t+1}$ in one time-step. Thus, the work done by the control is approximate as $P_{S t}=u_{S t}\left(x_{S t+1}-x_{S t}\right)$. That is to say, the control energy cost is $\left|P_{S t}\right|$. By that analogy, one can obtain (33).

For convenience, stochastic disturbance $d_{r}$ is not considered. Control parameters are the same as Case 1. Assume the control is activated at $\tau=10$ and the total simulation time is $\tau=50$. Control responses under different fractional-order $\lambda$ are shown in Figure 10. It is obvious from the figure that the trajectories converge faster with the increase of fractionalorder $\lambda$. FNFTSMC are more sensitive than INFTSMC when control is on. And it can also achieve a better "soft landing" to the desired trajectory. However, the increase of $\lambda$ can increase the possibility of chattering and power cost (Table 1). Anyway, compared with integer-order control, both the standard deviation of input and mean power of FNFTSMC are much less than those of INFTSMC. During control parameters selection, we should make reasonable trade-offs between convergence speed, chattering, and power cost. However, this is beyond the scope of our research.

\section{Conclusions}

This paper presents the chaotic dynamics of a doubly clamped microbeam actuated by two symmetrical electrodes. Different from the research by Haghighi and Markazi [16], this 

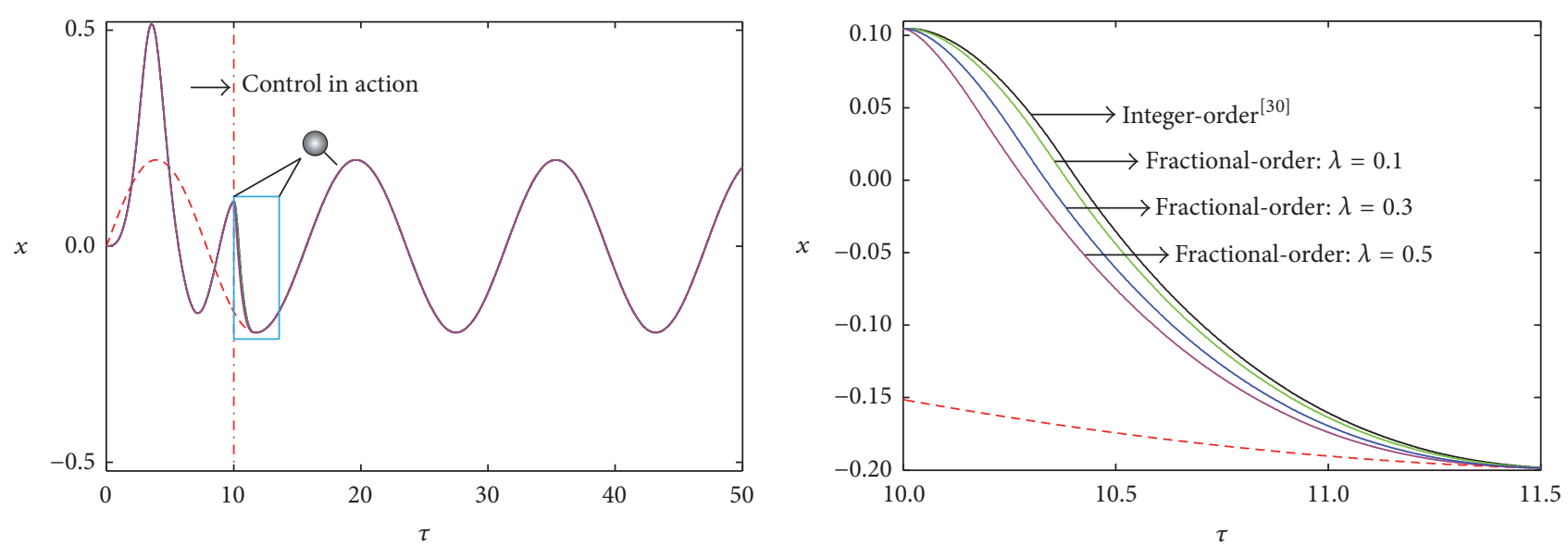

(a)
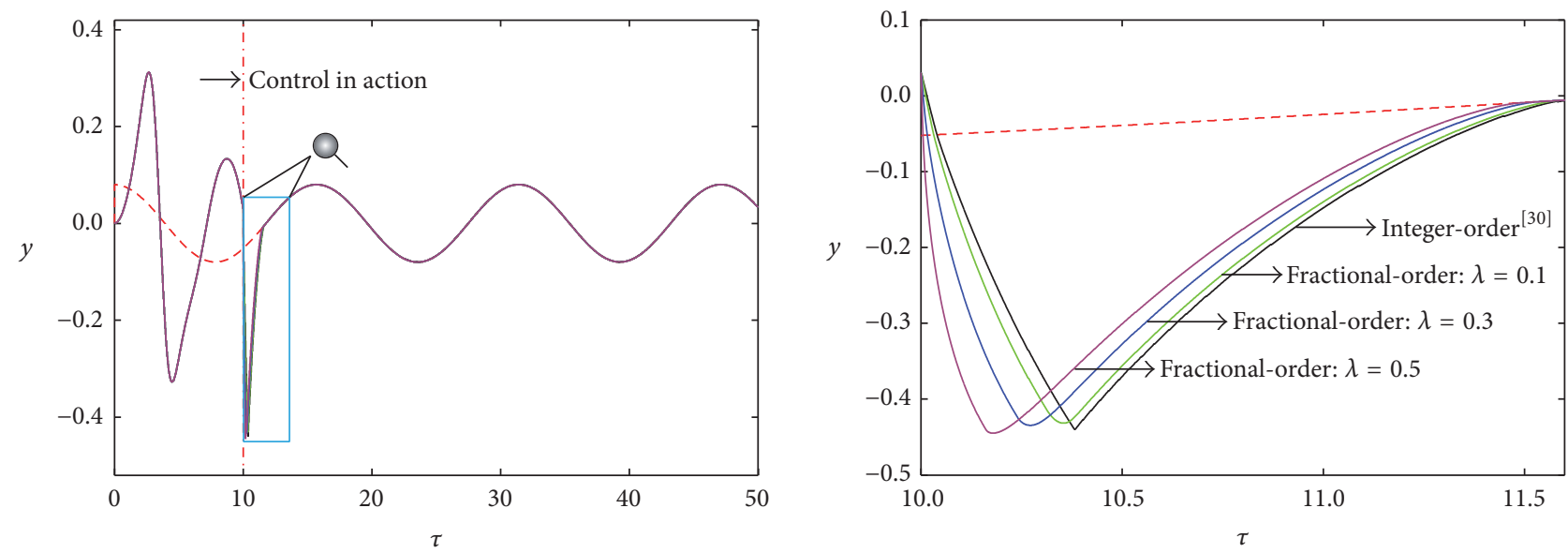

(b)

FIGURE 10: Control responses under different fractional-order $\lambda$ : (a) displacement response; (b) velocity response.

TABle 1: Performance of integer-order and fractional-order controllers.

\begin{tabular}{lccc}
\hline Controller & Order $\lambda$ & $\begin{array}{c}\text { Standard } \\
\text { deviation of } \\
\text { input } u\end{array}$ & Mean power $P$ \\
\hline INFTSMC & 0 & 1.4726 & 0.0847 \\
FNFTSMC & 1 & 0.1639 & 0.0089 \\
& 3 & 0.1961 & 0.0091 \\
& 5 & 0.2726 & 0.0094 \\
\hline
\end{tabular}

study mainly focuses on system dynamics when this MEMS resonator includes only one stable origin center. Through simulation results, we find, under primary resonance condition, that the resonator may exhibit chaotic behaviors as well. For the suppression of chaos, a novel control strategy, composed of an integer-order nonsingular fast terminal sliding surface with a fractional-order switching law, is proposed and shows its effectiveness in controlling chaotic motion. Besides, the existence of uncertainty and external and stochastic disturbance also verifies its strong robustness. Moreover, a qualitative analysis on the selection of fractionalorder is also carried out. Two statistical functions, standard deviation of input and mean power, are used to estimate the degree of chattering and energy cost. Simulation results (Figure 10 and Table 1) reveal that the increase of fractionalorder is accompanied by relatively higher chattering and bigger energy cost, but much faster convergence.

This study develops a fractional-order sliding mode control method for chaos control of MEMS resonator. The feasibility is numerically verified by comparing it with an integer-order sliding mode control [30]. However, control properties with different control parameters still need to be investigated in order to find a group of optimal parameters. Future work could focus on control parameter optimization by applying some optimization theories.

\section{Competing Interests}

The authors declare that there is no conflict of interests regarding the publication of this paper. 


\section{Acknowledgments}

The work was supported by the National Natural Science Foundation of China (Grants nos. 11372210, 11302149), Tianjin Research Program of Application Foundation and Advanced Technology (Grant no. 15JCQNJC05000), Innovation Team Training Plan of Tianjin Universities and Colleges (TD125043), Tianjin College Foundation of Development of Science and Technology (20130408), and Development Foundation of Tianjin University of Technology and Education (KJ11-06).

\section{References}

[1] M. I. Younis, MEMS Linear and Nonlinear Statics and Dynamics, Springer, New York, NY, USA, 2011.

[2] F. Abdul Rahim and M. I. Younis, "Control of bouncing in MEMS switches using double electrodes," Mathematical Problems in Engineering, vol. 2016, Article ID 3479752, 10 pages, 2016.

[3] M. I. Younis, E. M. Abdel-Rahman, and A. Nayfeh, "A reducedorder model for electrically actuated microbeam-based MEMS," Journal of Microelectromechanical Systems, vol. 12, no. 5, pp. 672-680, 2003.

[4] J. Liu, D. T. Martin, T. Nishida, L. N. Cattafesta III, M. Sheplak, and B. P. Mann, "Harmonic balance nonlinear identification of a capacitive dual-backplate MEMS microphone," Journal of Microelectromechanical Systems, vol. 17, no. 3, pp. 698-708, 2008.

[5] A. C. J. Luo and F. Y. Wang, "Chaotic motion in a micro-electromechanical system with non-linearity from capacitors," Communications in Nonlinear Science and Numerical Simulation, vol. 7, no. 1-2, pp. 31-49, 2002.

[6] B. E. DeMartini, H. E. Butterfield, J. Moehlis, and K. L. Turner, "Chaos for a microelectromechanical oscillator governed by the nonlinear mathieu equation," Journal of Microelectromechanical Systems, vol. 16, no. 6, pp. 1314-1323, 2007.

[7] S. Hu and A. Raman, "Chaos in atomic force microscopy," Physical Review Letters, vol. 96, no. 3, Article ID 036107, 2006.

[8] S. Zhankui and K. Sun, "Nonlinear and chaos control of a microelectro-mechanical system by using second-order fast terminal sliding mode control," Communications in Nonlinear Science and Numerical Simulation, vol. 18, no. 9, pp. 2540-2548, 2013.

[9] C.-C. Wang, N.-S. Pai, and H.-T. Yau, "Chaos control in AFM system using sliding mode control by backstepping design," Communications in Nonlinear Science and Numerical Simulation, vol. 15, no. 3, pp. 741-751, 2010.

[10] M. Ashhab, M. V. Salapaka, M. Dahleh, and I. Mezic, "Melnikov-based dynamical analysis of microcantilevers in scanning probe microscopy," Nonlinear Dynamics, vol. 20, no. 3, pp. 197-220, 1999.

[11] K. Yamasue and T. Hikihara, "Control of microcantilevers in dynamic force microscopy using time delayed feedback," Review of Scientific Instruments, vol. 77, no. 5, Article ID 053703, 2006.

[12] F. R. Chavarette, J. M. Balthazar, J. L. P. Felix, and M. Rafikov, “A reducing of a chaotic movement to a periodic orbit, of a microelectro-mechanical system, by using an optimal linear control design," Communications in Nonlinear Science and Numerical Simulation, vol. 14, no. 5, pp. 1844-1853, 2009.

[13] M. F. P. Polo, M. P. Molina, and J. G. Chica, "Chaotic dynamic and control for micro-electro-mechanical systems of massive storage with harmonic base excitation," Chaos, Solitons and Fractals, vol. 39, no. 3, pp. 1356-1370, 2009.

[14] A. M. Tusset, J. M. Balthazar, D. G. Bassinello, J. Pontes, and J. L. Felix, "Statements on chaos control designs, including a fractional order dynamical system, applied to a "MEMS" combdrive actuator," Nonlinear Dynamics, vol. 69, no. 4, pp. 1837-1857, 2012.

[15] A. Seleim, S. Towfighian, E. Delande, E. Abdel-Rahman, and G. Heppler, "Dynamics of a close-loop controlled MEMS resonator," Nonlinear Dynamics, vol. 69, no. 1-2, pp. 615-633, 2012.

[16] H. S. Haghighi and A. H. D. Markazi, "Chaos prediction and control in MEMS resonators," Communications in Nonlinear Science and Numerical Simulation, vol. 15, no. 10, pp. 3091-3099, 2010.

[17] H.-T. Yau, C.-C. Wang, C.-T. Hsieh, and C.-C. Cho, "Nonlinear analysis and control of the uncertain micro-electro-mechanical system by using a fuzzy sliding mode control," Computers \& Mathematics with Applications, vol. 61, no. 8, pp. 1912-1916, 2011.

[18] M. P. Aghababa, "Chaos in a fractional-order micro-electromechanical resonator and its suppression," Chinese Physics $B$, vol. 21, no. 10, Article ID 100505, 2012.

[19] I. Podlubny, Fractional Differential Equations: An Introduction to Fractional Derivatives, Fractional Differential Equations, to Methods of Their Solution and some of Their Applications, Academic press, San Diego, Calif, USA, 1998.

[20] M. A. Rahimian and M. S. Tavazoei, "Stabilizing fractionalorder PI and PD controllers: an integer-order implemented system approach," Proceedings of the Institution of Mechanical Engineers. Part I: Journal of Systems and Control Engineering, vol. 224, no. 8, pp. 893-903, 2010.

[21] B. Boudjehem and D. Boudjehem, "Fractional order controller design for desired response," Proceedings of the Institution of Mechanical Engineers, Part I: Journal of Systems and Control Engineering, vol. 227, no. 2, pp. 243-251, 2013.

[22] M. Ö. Efe, "Fractional order sliding mode control with reaching law approach," Turkish Journal of Electrical Engineering and Computer Sciences, vol. 18, no. 5, pp. 731-747, 2010.

[23] M. P. Aghababa, "A fractional-order controller for vibration suppression of uncertain structures," ISA Transactions, vol. 52, no. 6, pp. 881-887, 2013.

[24] S. Dadras and H. R. Momeni, "Control of a fractional-order economical system via sliding mode," Physica A: Statistical Mechanics and its Applications, vol. 389, no. 12, pp. 2434-2442, 2010.

[25] I. Kheirizad, A. A. Jalali, and K. Khandani, "Stabilization of fractional-order unstable delay systems by fractional-order controllers," Proceedings of the Institution of Mechanical Engineers, Part I: Journal of Systems and Control Engineering, vol. 226, no. 9, pp. 1166-1173, 2012.

[26] M. P. Aghababa, "A novel terminal sliding mode controller for a class of non-autonomous fractional-order systems," Nonlinear Dynamics, vol. 73, no. 1-2, pp. 679-688, 2013.

[27] M. Ö. Efe, "A sufficient condition for checking the attractiveness of a sliding manifold in fractional order sliding mode control," Asian Journal of Control, vol. 14, no. 4, pp. 1118-1122, 2012.

[28] Y. Tang, X. Zhang, D. Zhang, G. Zhao, and X. Guan, "Fractional order sliding mode controller design for antilock braking systems," Neurocomputing, vol. 111, pp. 122-130, 2013. 
[29] E. Maani Miandoab, H. N. Pishkenari, A. Yousefi-Koma, and F. Tajaddodianfar, "Chaos prediction in MEMS-NEMS resonators," International Journal of Engineering Science, vol. 82, pp. 74-83, 2014.

[30] L. Yang and J. Yang, "Nonsingular fast terminal sliding-mode control for nonlinear dynamical systems," International Journal of Robust and Nonlinear Control, vol. 21, no. 16, pp. 1865-1879, 2011.

[31] C. Li and W. Deng, "Remarks on fractional derivatives," Applied Mathematics and Computation, vol. 187, no. 2, pp. 777-784, 2007.

[32] Y. Li, Y. C. Chen, and I. Podlubny, "Stability of fractionalorder nonlinear dynamic systems: Lyapunov direct method and generalized Mittag-Leffler stability," Computers \& Mathematics with Applications, vol. 59, no. 5, pp. 1810-1821, 2010.

[33] Y. Li, Y. Chen, and I. Podlubny, "Mittag-Leffler stability of fractional order nonlinear dynamic systems," Automatica, vol. 45, no. 8, pp. 1965-1969, 2009.

[34] A. Oustaloup, F. Levron, B. Mathieu, and F. M. Nanot, "Frequency-band complex noninteger differentiator: characterization and synthesis," IEEE Transactions on Circuits and Systems I: Fundamental Theory and Applications, vol. 47, no. 1, pp. 25-39, 2000.

[35] D. Y. Xue, C. N. Zhao, and Y. Q. Chen, "A modified approximation method of fractional order system," in Proceedings of the IEEE International Conference on Mechatronics and Automation (ICMA '06), pp. 1043-1048, IEEE Computer Society, Luoyang, China, June 2006.

[36] R. M. C. Mestrom, R. H. B. Fey, J. T. M. van Beek, K. L. Phan, and H. Nijmeijer, "Modelling the dynamics of a MEMS resonator: simulations and experiments," Sensors and Actuators A: Physical, vol. 142, no. 1, pp. 306-315, 2008.

[37] J. Han, Q. Zhang, and W. Wang, "Design considerations on large amplitude vibration of a doubly clamped microresonator with two symmetrically located electrodes," Communications in Nonlinear Science and Numerical Simulation, vol. 22, no. 1-3, pp. 492-510, 2015.

[38] J. Han, Q. Zhang, and W. Wang, "Static bifurcation and primary resonance analysis of a MEMS resonator actuated by two symmetrical electrodes," Nonlinear Dynamics, vol. 80, no. 3, pp. 1585-1599, 2015.

[39] F. M. Alsaleem, M. I. Younis, and L. Ruzziconi, "An experimental and theoretical investigation of dynamic pull-in in MEMS resonators actuated electrostatically," Journal of Microelectromechanical Systems, vol. 19, no. 4, pp. 794-806, 2010.

[40] A. H. Nayfeh, M. I. Younis, and E. M. Abdel-Rahman, "Dynamic pull-in phenomenon in MEMS resonators," Nonlinear Dynamics, vol. 48, no. 1-2, pp. 153-163, 2007.

[41] A. H. Davaie-Markazi and H. Sohanian-Haghighi, "Chaos analysis and control in AFM and MEMS resonators," in Chaotic Systems, pp. 119-134, InTech, 2011.

[42] M. P. Aghababa, "A switching fractional calculus-based controller for normal non-linear dynamical systems," Nonlinear Dynamics, vol. 75, no. 3, pp. 577-588, 2014.

[43] M. P. Aghababa, "Finite-time chaos control and synchronization of fractional-order nonautonomous chaotic (hyperchaotic) systems using fractional nonsingular terminal sliding mode technique," Nonlinear Dynamics, vol. 69, no. 1-2, pp. 247-261, 2012. 


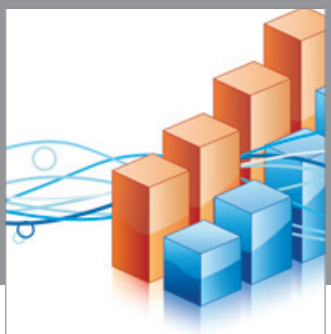

Advances in

Operations Research

vatem alat4

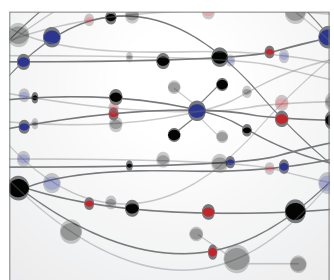

\section{The Scientific} World Journal

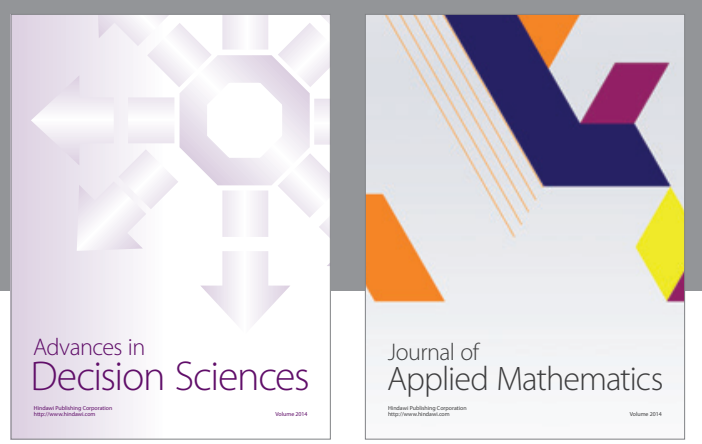

Algebra

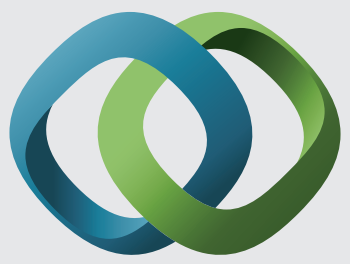

\section{Hindawi}

Submit your manuscripts at

https://www.hindawi.com
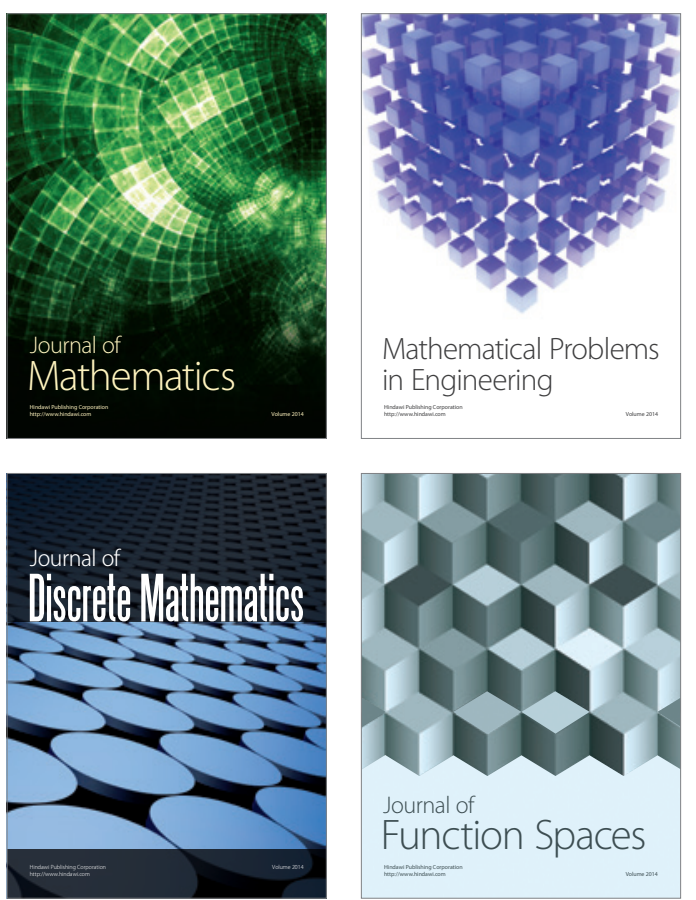

Mathematical Problems in Engineering
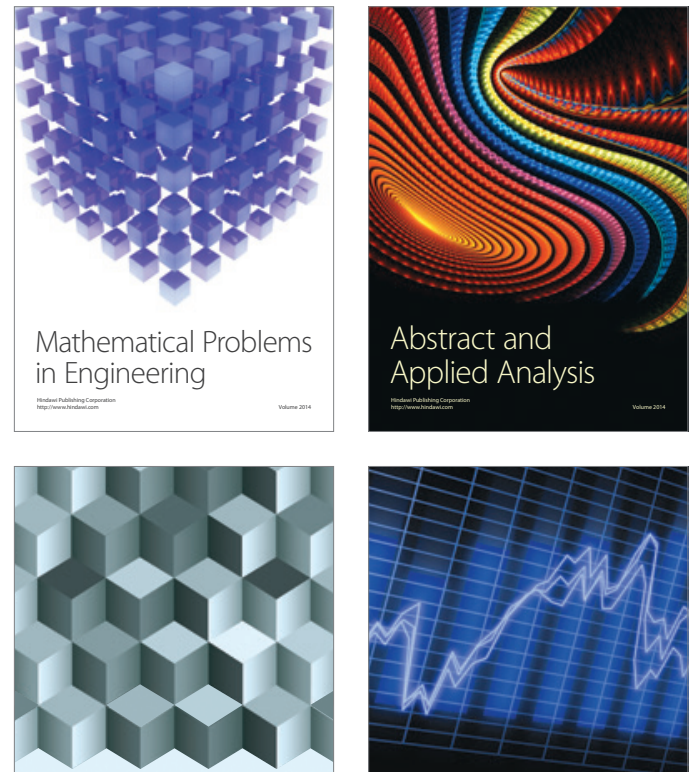

Journal of

Function Spaces

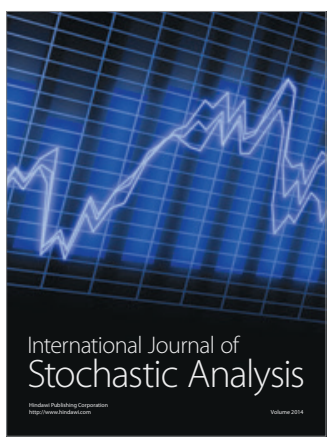


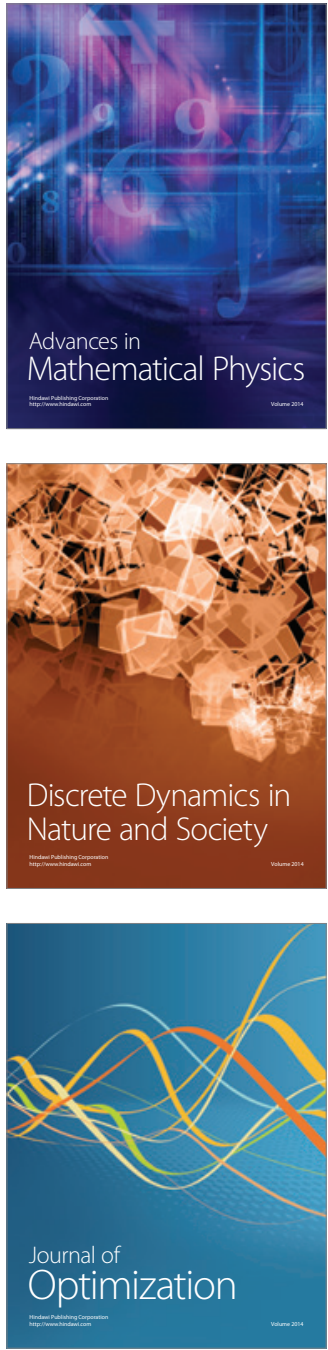